\title{
Role of adatom defects in the adsorption of polyaromatic hydrocarbons on metallic substrates
}

Dushanthi Dissanayake ${ }^{1}$, Yarra Hassan ${ }^{2}$, Emilian Tuca ${ }^{3}$, and Irina Paci ${ }^{2}$

${ }^{1}$ Department of Biochemistry and Microbiology, University of Victoria, Victoria, British Columbia, Canada

${ }^{2}$ Department of Chemistry, University of Victoria, Victoria, British Columbia, Canada

${ }^{3}$ Department of Chemistry, Irving K. Barber Faculty of Science, University of British Columbia, Okanagan, British Columbia, Canada

\begin{abstract}
The adsorption of polyaromatic hydrocarbons (PAHs) on metallic substrates has been of interest in the field of optoelectronics due to the possibility of designing complex materials with tunable properties through surface functionalization with organic molecules. Much of the modelling research in this field has focused on perfectly symmetrical (idealized) substrates. Limited research has investigated the effect of substrate irregularities, such as adatoms, on the binding of PAHs onto substrates. Here, we examine how the presence of substrate-bound adatoms affects the binding of coronene and hexahelicene monomers and dimers onto $\mathrm{Au}(111)$ and $\mathrm{Cu}(111)$ substrates using a density functional theory approach. We found that helicene monomers were more effectively able to adapt to the presence of the adatoms than coronene by coiling around the adatoms. Whereas upon adsorption on an ideal (111) surface, coronene can establish significantly stronger dispersive interactions than helicenes, adatom defects reverse the trend. For helicenes, the degree of flattening near the surface and molecular coiling were strongly influenced by the extent of the adatom defect, as a result of the interplay between the molecule's drive to maximize overlap with the underlying surface and the enhanced reactivity of lower-coordinated adatoms.
\end{abstract}

Key words: hexahelicene, adatoms, coinage metals, polyaromatic hydrocarbons, chirality, substrate defects, density functional theory 


\section{Introduction}

Over the past two decades, interest in the self-assembly of organic molecules on solid metal substrates has grown considerably, driven by an opportunity to develop inexpensive, tunable organic optoelectronic devices. ${ }^{1-11}$ Such systems synergistically combine the electrical conductivity of the metal with the highly tunable properties of organic molecules, enabling the potential for new properties and functionalities not possessed by either material separately., ${ }^{9,2}$ The high sensitivity of the electronic and optical properties of the material to the choice of adsorbate highlights the advantage of optimizing the adsorbate/substrate pair when designing organic electronic materials. ${ }^{12}$ The advancement of Organic Light Emission Diodes (OLEDs) and Organic Field Effect Transistors (OFETs) to the market highlights the promise and versatility of organic optoelectronic devices. ${ }^{3}$ However, there is still much work to be done in developing a molecular-level understanding of the chemical and electronic nature of the interface, the impact of the identity of the substrate and adsorbate pair, and the distinct substrate/adsorbate interactions. ${ }^{3}$ The large size of the minimum representative unit and the complex interactions (e.g. covalent bonding, van der Waals, charge transfer, Pauli repulsion) in such systems makes modelling the structure and stability of such systems challenging. ${ }^{12}$ Recent progress in computational capability has made feasible the application of computational techniques involving quantum approaches, such as density functional theory (DFT) and ab initio molecular dynamics (AIMD), to these systems.

Helicenes are an interesting class of adsorbates due to their molecular chirality, the formation of self-assembled monolayers upon adsorption on metallic substrates, the chiral polymorphism of the assembled patterns, and their intriguing optical properties. ${ }^{1,13-15}$ Structurally, they are highly-conjugated, ortho-fused, chiral, aromatic hydrocarbons. ${ }^{13,16}$ The chirality of helicenes, despite the lack of a chiral center, arises from the fusion of benzene rings in the ortho position, such that they adopt a chiral helical topology when the backbone is large enough to encounter steric repulsion of the two ends. ${ }^{1,16}$ As chiral PAHs, helicenes can facilitate asymmetric and 
enantioselective reactions, including those of biological importance, and have been shown ability to transfer chirality to supramolecular systems through directed self-assembly. ${ }^{17-19}$

Due to their helical backbones, helicenes generally bind to the substrate through 2-4 terminal rings, despite significant dispersive interaction of the entire backbone to the surface. ${ }^{1,13,20}$ As a result, some of the molecular structure flattens when adsorbed, and subsequent deformations propagate through the entire backbone. A good understanding of the interaction of the terminal rings with the surface is thus essential in developing insight into stereochemical recognition within the self-assembled structure. ${ }^{13}$ On the other hand, the structure of coronene (denoted below as [6]C) can also be interpreted as an ortho-fused 6-ring PAH (analogous with hexahelicene $[6] \mathrm{H})$, but with the ends fused together. Coronene exhibits a planar structure, maximizing dispersive interactions on flat, ideal metallic substrates. This, combined with the similar footprint of [6]C and [6] $\mathrm{H}$, makes [6]C a good reference for studies pertaining to helicene adsorption on metal substrates. ${ }^{1}$ As a large planar PAH, coronene has been well studied in its own right as a model for graphene sheets and related nanomaterials. ${ }^{21-23}$

Considerable work has been conducted on the self-assembly behaviour of helicenes on coinage metal substrates, ${ }^{1,13,24-31}$ with much of the discussion focusing on adsorption on perfectly symmetrical (idealized) substrates. Our group has recently performed computational investigations of the behaviour of helicene monomers and stacked dimers on ideal (111) coinage metal substrates. ${ }^{1}$ We found that single helicene molecules bound more strongly to $\mathrm{Cu}(111)$ than $\operatorname{Ag}(111)$ and $\mathrm{Au}(111)$ substrates, and that [6]C was able to bind more efficiently due to greater dispersive interactions with the underlying substrate. A strong flattening of the three surface-adjacent rings was observed, in agreement with work by Ernst et al. ${ }^{13}$ Deposition of further molecules led to preferentially homochiral arrangements in both lateral and stacked configurations, with stacked configurations deforming the surface-supported molecule in the most favorable binding configurations. Similar behaviour was observed for [5] H, [6]H, and [7]H. The findings of our study served to indicate, in conjunction with experimental findings on monolayer and multilayer chirality behaviour, that small changes at the single molecule level 
may have vast implications for the behavior of the mixed monolayer. The question of how the behavior of helicenes changes in the vicinity of surface defects thus becomes an important one to address. In order to better understand interactions of helicenes on realistic substrates, coinage metal substrates were decorated with metallic adatoms. Here, we investigate the binding of [6] $\mathrm{H}$ monomers and dimers, as well as [6]C, on $\mathrm{Au}(111)$ and $\mathrm{Cu}(111)$ substrates with one to four adatoms, using computational chemistry modelling.

There has been some prior work on the role of adatoms in the adsorption of conjugated aromatic molecules onto metallic substrates. Bartels (2010) found that adatoms will diffuse onto the substrate even at temperatures as low as $200 \mathrm{~K}$, highlighting the likelihood of adatoms being present on deposition substrates, particularly on soft metals such as copper. ${ }^{32}$ Current understanding suggests that adatoms behave as coordination sites, with up to four ligands bound to one adatom, in a coordination geometry that generally reflects the symmetry of the underlying metal lattice (e.g. three-fold for fcc (111) substrates and two- or four-fold for fcc(100) substrates). ${ }^{32}$ On metal substrates, the nature of coordination bonds is less well understood than in solution phase, where the formal oxidation state of the metal adatoms can be defined. On metals, the coordination geometry is more complex because of the steric effect of the underlying surface, as well as due to its electronic effect: the metal substrate acts as an electron reservoir, allowing substrate coordination centers to donate, or potentially accept, charge without becoming charged themselves. Furthermore, metal adatoms situated atop bare metal substrates rest approximately $10 \%$ closer to the substrate than the substrate layer spacing, allowing the adatoms' charge to spread out into the substrate, leading to polarization of the adatom site. Modelling these fundamentally quantum effects requires use of quantum chemistry techniques, such as DFT, and large simulation sizes.

In the following pages, we report on our investigation of the effect of 1-4 adatom defects at $\mathrm{Au}(111)$ and $\mathrm{Cu}(111)$ surfaces on the adsorption of [6] H and [6]C, as well as any chirality aspects of [6]H adsorption. Overall, this work paves the way for a more accurate understanding 
of the self-assembly of organic molecules in real organic-metal systems and thus helps facilitate the path forward to developing more efficient optoelectronic devices in the future.

\section{Models and methods}

We consider the adsorption of $P$ - and $M$-[6]H on $\mathrm{Au}(111)$ and $\mathrm{Cu}(111)$ substrates functionalized with 1-4 adatoms. Helicene dimers, also discussed here for one- and three-adatom systems, will be denoted as $M M, P P$ or $M P$, based on the stereochemical designation of the two enantiomers. Gas phase structures of [6]C and [6]H optimized in DFT were used to create input geometries for the surface-supported systems. Figure 1 presents the optimized structures of the polyaromatic hydrocarbon adsorbates investigated in our work.

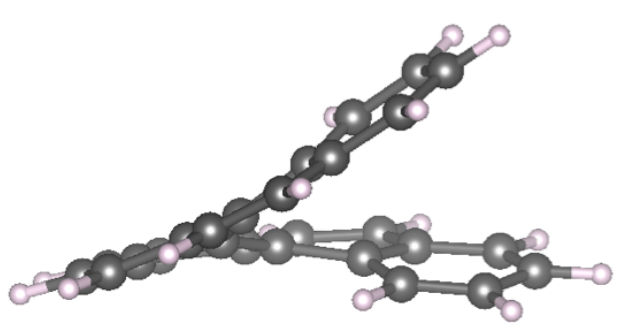

(a)

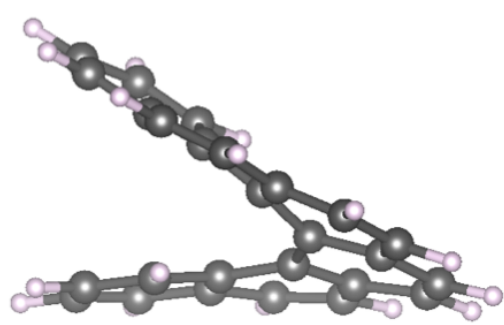

(b)

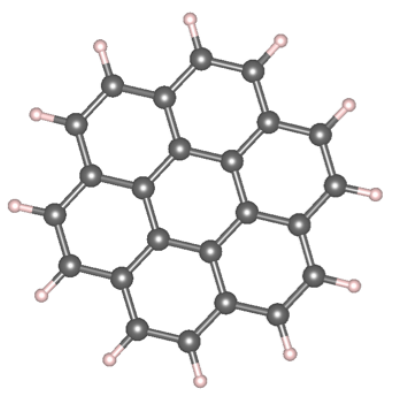

(c)

Figure 1. DFT-optimized structures of polyaromatic hydrocarbon adsorbates. The $\mathrm{C}$ atoms are shown in grey and the $\mathrm{H}$ atoms are shown in pink. Panels (a)-(c) show $P-[6] \mathrm{H}, M-[6] \mathrm{H}$, and $[6] \mathrm{C}$, respectively.

\section{Substrate details}

Adatoms were built upon the symmetric $\mathrm{Au}(111)$ and $\mathrm{Cu}(111)$ slabs used by Tuca and Paci previously. ${ }^{1}$ Periodic boundary conditions were used, with simulation cells of dimensions $x=y=$ $2.1 \mathrm{~nm}$ for $\mathrm{Au}$, and $1.8 \mathrm{~nm}$ for $\mathrm{Cu}$. A vacuum of $7 \mathrm{~nm}$ was added above the lattice in order to separate image slabs in the $z$ direction. Cell sizes were large enough so that intermolecular interactions between neighbouring images of the adsorbates were negligible. Each surface model had three layers of substrate atoms, with each layer having 56 atoms (168 atoms in total in the basal surface). The ideal substrates were then functionalized with 1-4 adatoms to create 
"defects". Initial locations for the adatoms were approximately centered above the surface, but occupying positions along the regular surface lattice, in nearest-neighbor configurations. Figure 2 presents the optimized structures of the slab surfaces with adatom defects investigated in our work. These substrate geometries were used in generating initial configurations for all systems. In all calculations, atoms in the bottom-most substrate layer were constrained to the optimized geometry of the bulk crystal structure, whereas the top two layers of atoms, as well as the adatoms, were allowed to reorganize and optimize. Simulation box sizes and substrate structures (except for the adatoms) were consistent across all systems.

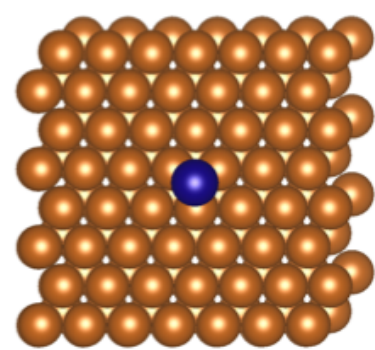

(a)

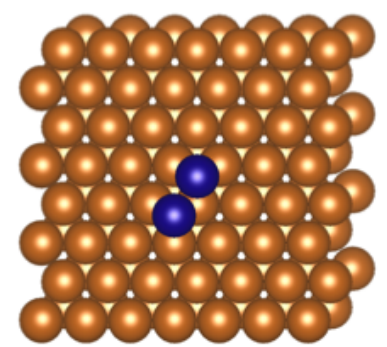

(b)

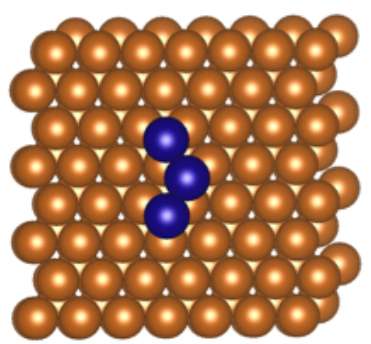

(c)

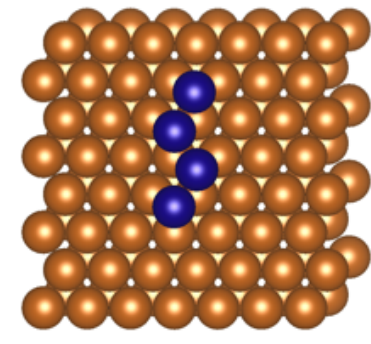

(d)

Figure 2. DFT-optimized structures of a $\mathrm{Cu}(111)$ substrate with adatom defects. Regular $\mathrm{Cu}$ substrate atoms are shown in orange. $\mathrm{Cu}$ adatoms are shown in blue. Substrate models with 1, 2, 3 , and 4 adatoms are shown in panels (a)-(d), respectively.

\section{Computational Method}

\section{Structural optimization of adsorbed helicenes and coronone}

The computational approach used in the current work follows the process presented in Ref. [1]. A PBE-TS/DZP approach was used, as implemented in the SIESTA 4.0 code. ${ }^{33-38}$ The binding energies reported below were calculated as the enthalpy change associated with the adsorption reaction. Bound systems are reported to have a negative binding energy. Basis set superposition errors (BSSE) were estimated and corrected using the counterpoise approach. ${ }^{39-41}$ Extending the confinement radius of the pseudoatomic orbitals (PAOs), as described by Chapman et al., ${ }^{42}$ has an effect similar to extending the basis set size, with larger confinement radii leading to diminishing BSSE. As before, a PAO energy shift of $1 \mathrm{mRy}$ (equivalent to $6 \AA$ for the cut-off radius of the carbon PAO with the smallest $\zeta$ ) was used. ${ }^{41}$ 
Dynamic behaviour of adsorbed monomers and dimers

In order to explore the configurational space around optimized structures, AIMD simulations were performed for representative systems, using the SIESTA molecular dynamics implementation. The Nosé thermostat was used to maintain a constant temperature throughout each simulation. The target temperature was set to $250.0 \mathrm{~K}$. 2000-4000 steps were performed with a timestep of $1.0 \mathrm{fs}$.

\section{Quantifying helicene deformation}

To estimate the degree of deformation of the helicene molecules upon surface adsorption, we calculated the height of each helicene ring from the surface adatom position. In the following discussion, the height of a ring is defined as the distance between the geometric center of the ring carbon backbone and the geometric center of the reference, projected on the $\mathrm{z}$ axis. On surfaces with one or more adatoms, the reference is the collection of all adatoms. In cases where no adatoms are present, the reference is the top-most atomic layer of the ideal surface. In other words, the reference layer is the top-most layer of the surface, whether this is saturated with atoms (ideal surface), or consists of just one or a few adatoms.

\section{Results and Discussion}

\section{Electronic structure}

Projected densities of states (PDOS) for the different participants in the binding process are shown in Figure 3. In general, for a bare surface, surface-atom PDOS depends on the site's coordination number and not on the number of adatoms present on the surface. PDOS calculated for sites with the same coordination numbers but on substrates with different adatom numbers, completely overlap with those shown in Figure 3(a). Depositing the adsorbate broadens a surface atom's PDOS, and the shift to lower energies indicates favourable binding, with a slightly stronger shift for $M$-[6]H than [6]C as evidenced in the lower panel of Figure 3(a). The effects of binding are more clearly shown in the carbon-atom PDOS plots in Figures 3(b)-(d). 

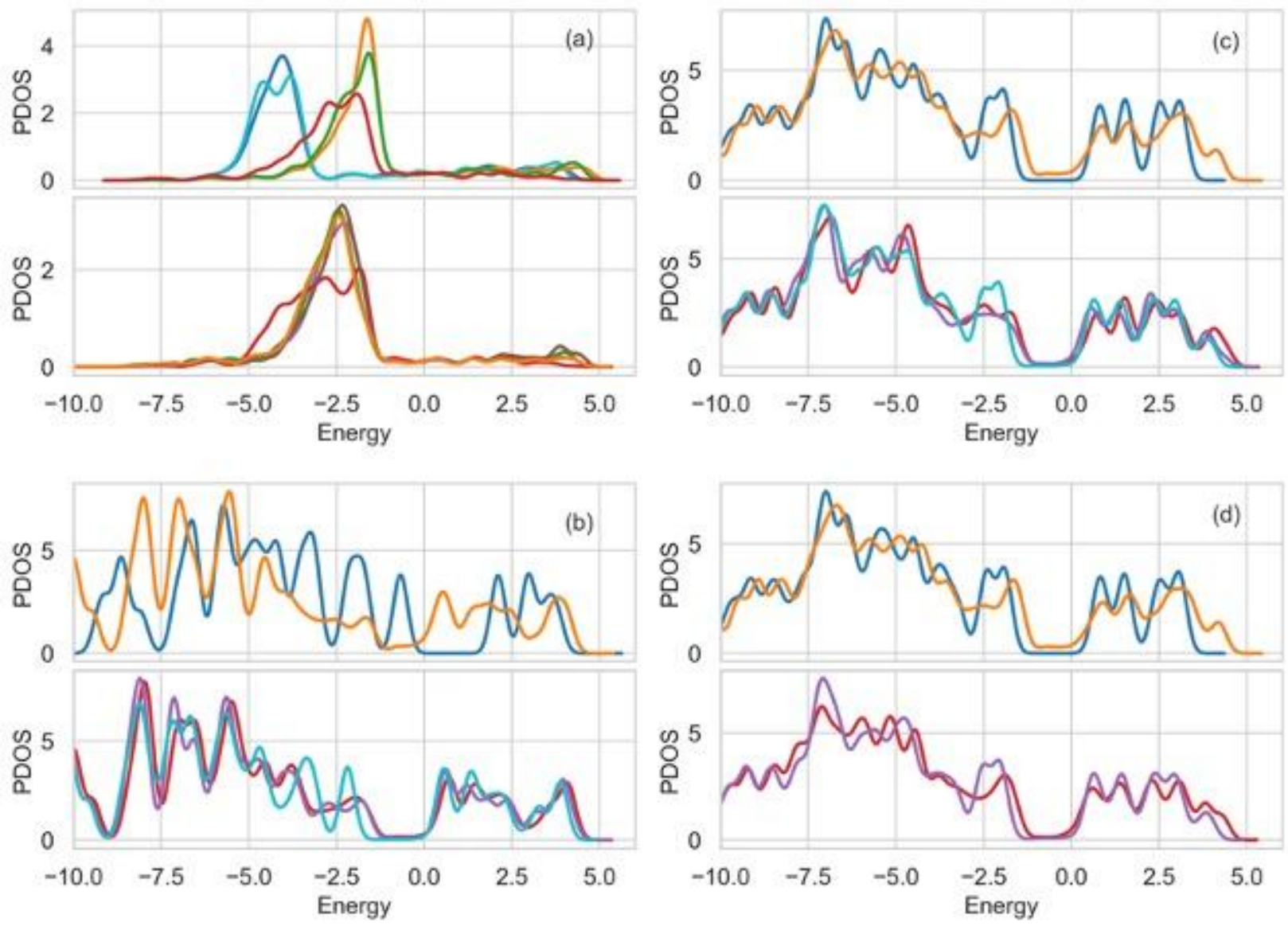

Figure 3. PDOS of various binding partners. In (a), adatom site PDOS are shown for the bare $\mathrm{Cu}$ and $\mathrm{Au}$ surface (top) and the bound $\mathrm{Cu}$ surface (bottom). Top (a) lines: $\mathrm{Cu}-\mathrm{CN} 4$ (Cu adatom with coordination number 4), $\mathrm{Cu}-\mathrm{CN} 5, \mathrm{Cu}-\mathrm{CN} 9, \mathrm{Au}-\mathrm{CN} 4$, and $\mathrm{Au}-\mathrm{CN} 5$ are shown in orange, green, red, blue, and cyan, respectively. Bottom (a): $\mathrm{Cu}-\mathrm{CN} 4$ is bound to [6]C (purple - 3 adatoms, green - 2 adatoms) and $P$-[6]H (orange - 3 adatoms, purple - 2 adatoms), and $\mathrm{Cu}-\mathrm{CN} 9$ bound to $[6] \mathrm{C}$ (red). In (b), (c), and (d), all-carbon atom PDOS are shown for [6]C, $M-[6] \mathrm{H}$ and $P-[6] \mathrm{H}$, respectively. In all three panels, data are shown for the unbound (gas phase) adsorbate (blue), adsorbate bound to ideal $\mathrm{Cu}(111)$ surface (orange), 2-adatom $\mathrm{Cu}$ (red), 3-adatom $\mathrm{Cu}$ (purple) and 3-adatom Au (cyan).

\section{Defect size effects}

Adatom defects have a considerable impact on the adsorption of PAHs. On an ideal (flat) surface, $[6] \mathrm{C}$ binds more strongly than either of the [6] H enantiomers to the substrate. Its planarity and 
extended conjugation maximize dispersive molecule-surface interactions for [6]C (see Figure 4(a) for ring height analysis and Table 1 for binding energies, both consistent with Ref. [1]). Note that the distance reference for an ideal surface is shifted downwards by one layer, relative to surfaces with adatoms, as described in the Models section. The strong surface interaction is reflected in the major impact of surface binding on the carbon-atom PDOS for [6]C [Figure 4(b)]. Helicene molecules are non-planar and thus experience weaker binding to ideal metallic substrates, despite extended conjugation and a footprint similar to that of [6]C. Helicenes generally flatten slightly in the vicinity of the ideal surface: In [6] H, up to 4 rings become parallel to the surface upon adsorption, thus maximizing dispersive binding interactions, as shown in blue in Figures 5(b) and (c) and discussed at length in Ref. [1]. Among coinage metal substrates, PAHs bind more strongly to $\mathrm{Cu}(111)$ than $\mathrm{Ag}(111)$ or $\mathrm{Au}(111) .{ }^{1,43}$ This is primarily attributed to the lower-energy location of the metallic d-band in the latter metals [reflected in Figure 3(a), for example], which places more of the resulting antibonding orbitals of the complex below the Fermi level. ${ }^{44,45}$ 

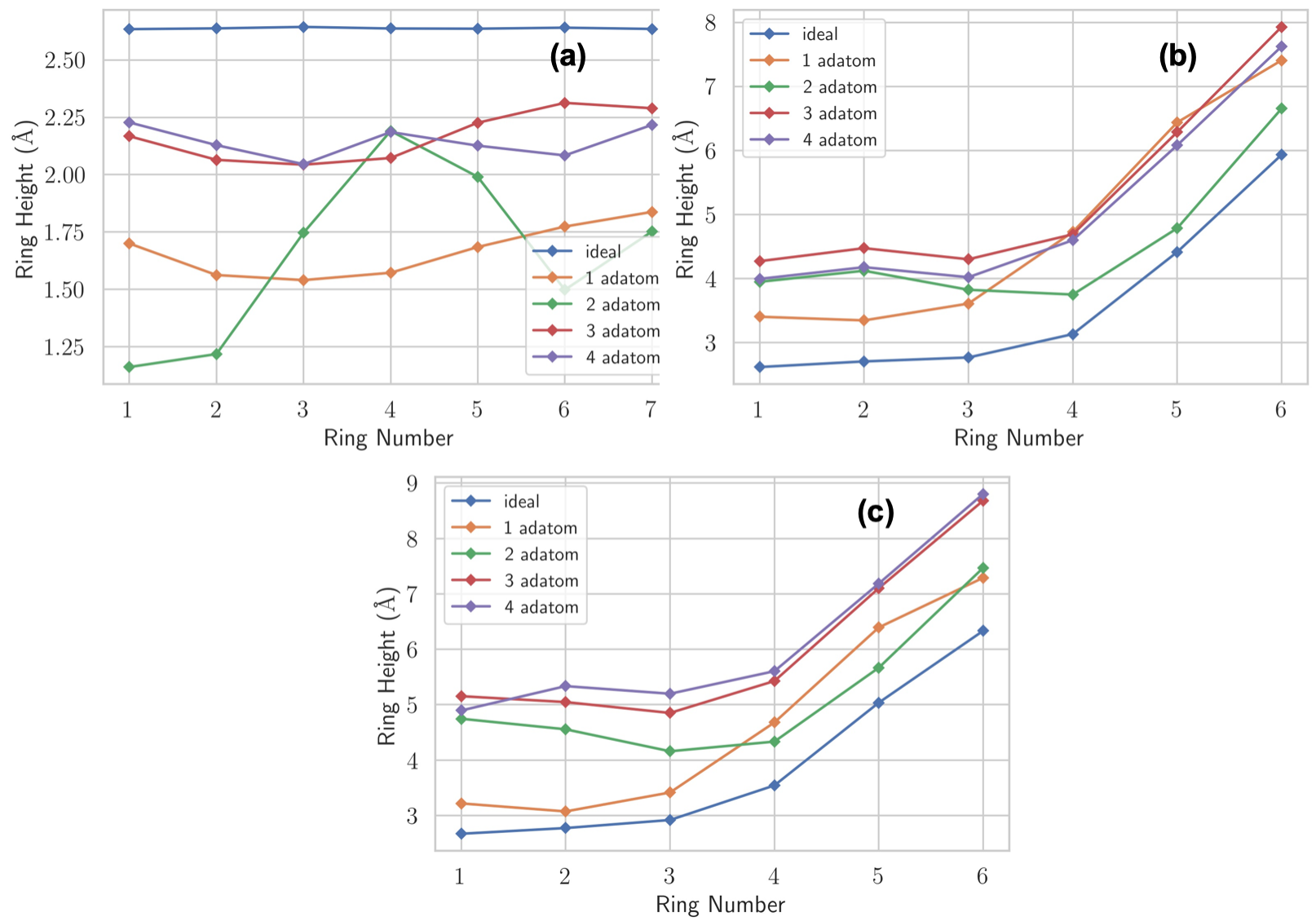

Figure 4. Successive ring heights relative to the topmost layer of $\mathrm{Cu}$ surface atoms for the various adatom configurations. Results for [6]C, $M$-[6]H and $P-[6] \mathrm{H}$ are shown in panels (a)-(c), respectively. Helicene rings were numbered starting with the terminal ring closest to the surface. Only peripheral rings are numbered for coronene, starting with any ring. Blue diamonds indicate ring heights for molecules on ideal surfaces, whereas orange, green, red, and purple diamonds are for substrates with 1-4 adatoms, respectively. Ring heights are relative to the adatom center of mass in the adatom surface, and the average $z$ coordinate of the upper surface layer for the ideal substrates. Lines between symbols were added to the figure for ease of identification.

Table 1. Binding energies of substrate-adsorbed polyaromatic hydrocarbons on $\mathrm{Au}(111)$ and $\mathrm{Cu}(111)$ substrates with 0-4 adatoms.

Binding energy (eV)

\begin{tabular}{lllllllllllll}
\hline & \multicolumn{2}{c}{$\mathbf{E}_{0 \mathrm{~A}}$} & & \multicolumn{2}{c}{$\mathbf{E}_{1 \mathrm{~A}}$} & & \multicolumn{2}{c}{$\mathbf{E}_{2 \mathrm{~A}}$} & & \multicolumn{2}{c}{$\mathbf{E}_{3 \mathrm{~A}}$} & \multicolumn{2}{c}{$\mathbf{E}_{4 \mathrm{~A}}$} \\
\hline Adsorbate & $\mathrm{Au}$ & $\mathrm{Cu}$ & $\mathrm{Au}$ & $\mathrm{Cu}$ & $\mathrm{Au}$ & $\mathrm{Cu}$ & $\mathrm{Au}$ & $\mathrm{Cu}$ & $\mathrm{Au}$ & $\mathrm{Cu}$
\end{tabular}




$\begin{array}{ccccccccccc}M-[6] \mathrm{H} & -1.29 & -1.78 & -1.15 & -1.84 & -1.24 & -2.22 & -1.38 & -2.42 & -1.12 & -2.47 \\ P-[6] \mathrm{H} & -1.28 & -1.73 & -1.04 & -1.82 & -1.14 & -2.32 & -1.47 & -2.49 & -1.13 & -2.45 \\ {[6] \mathrm{C}} & -1.58 & -2.12 & -0.73 & -1.72 & -1.19 & -2.24 & -1.10 & -2.03 & -1.09 & -2.30\end{array}$

Upon introducing a single adatom defect, the planarity advantage of [6]C is lost. [6]C bows slightly over the adatom, but the adatom's presence leads to significantly diminished dispersive interactions between the adsorbate and the metallic surface, as shown in Table 1 and Figure 4(a). Upon adsorption, the molecule remained nearly parallel to the surface (as on the $\mathrm{Cu}$ surface, Figure 5) or slightly tilted (as on the Au surface, Figure 6). Analysis of the density of states projected onto the collection of $\mathrm{C}$ atoms shows much weaker restructuring of the adsorbate density of states in the 1-adatom surface than in the flat surface case, consistent with weaker binding.

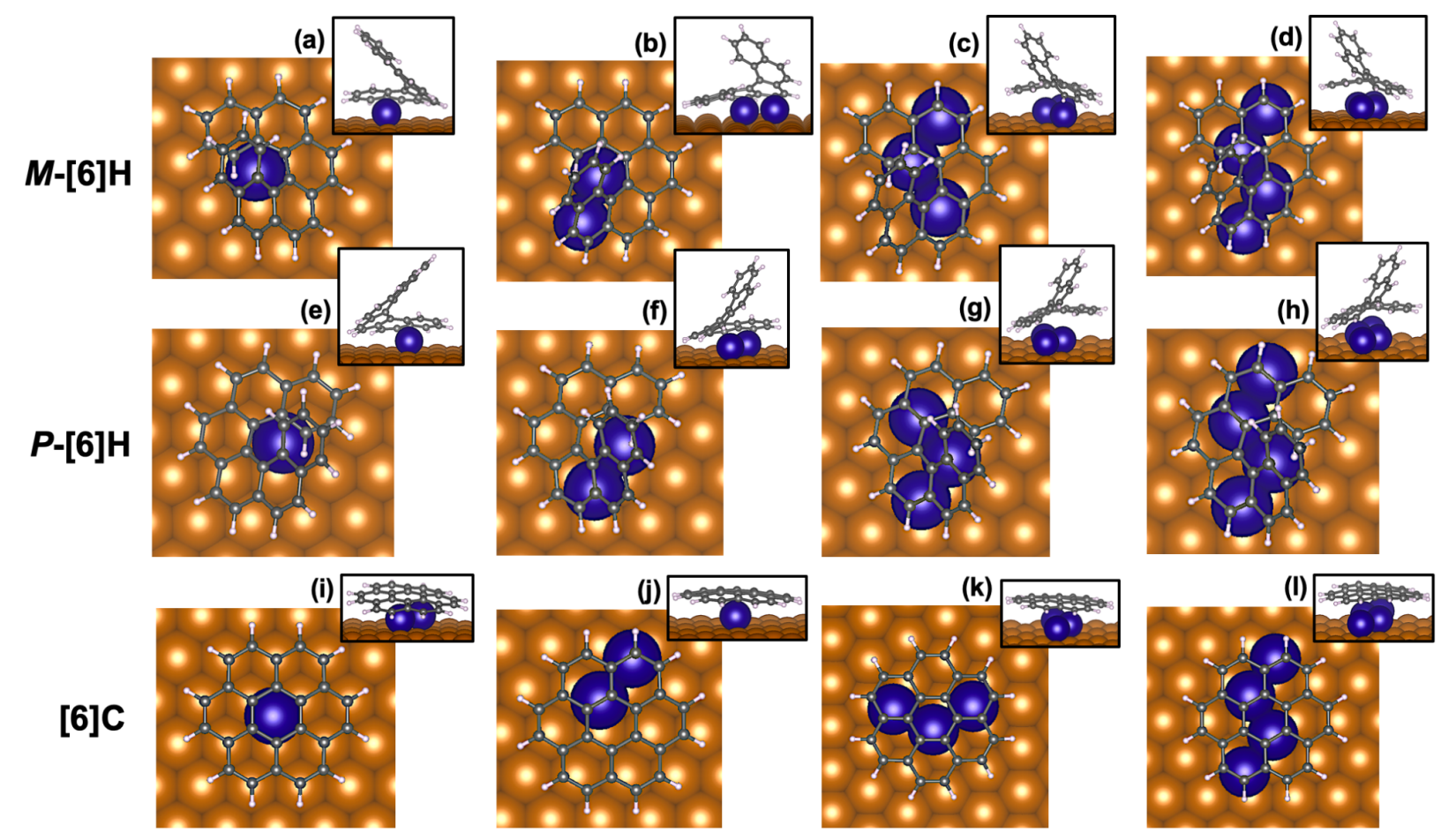

Figure 5. $M-[6] \mathrm{H}, P-[6] \mathrm{H}$, and [6]C adsorbed on $0,1,2,3$, and 4 adatom $\mathrm{Cu}$ substrates. Regular $\mathrm{Cu}$ substrate atoms are shown in orange. $\mathrm{Cu}$ adatoms are shown in blue. The full-width image is the top view of the system while the inset image is the side view. $M$-[6]H configurations adsorbed on surfaces with 0-4 adatoms are shown in (a)-(d), respectively. $P$-[6]H configurations 
adsorbed on 0-4-adatom $\mathrm{Cu}$ substrates are shown in (e)-(h), respectively. [6]C structures adsorbed on 0-4-adatom $\mathrm{Cu}$ substrates are shown in (i)-(1), respectively.

Further addition of adatoms restores much of the surface interaction for the $[6] \mathrm{C}-\mathrm{Cu}$ complex, as the highly reactive adatoms interact favourably with the nearby [6]C rings. [6]C's footprint is larger than the adatom range in all cases, and thus the molecule-surface interaction is a combination of the strong bonding of reactive adatoms and the center of the molecule, and the interaction of the molecular edges with the underlying extended surface. This effect is particularly evident in the case of the 2-adatom surface, which provokes the strongest molecular deformation. For 2-4 adatoms, the distance between adatoms and the rings above the adatoms approaches $2.2 \AA$, which is significantly closer than the average distances between [6]C and an ideal surface $(\sim 2.7 \AA)$. Molecular deformation is much less extensive over the 3-and 4-adatom substrates, where the more extended adatom structure is able to support a larger part of the molecular structure, and the energetic penalty of molecular deformation is not justified (see Figures 4(a) and 5.

M-[6]H
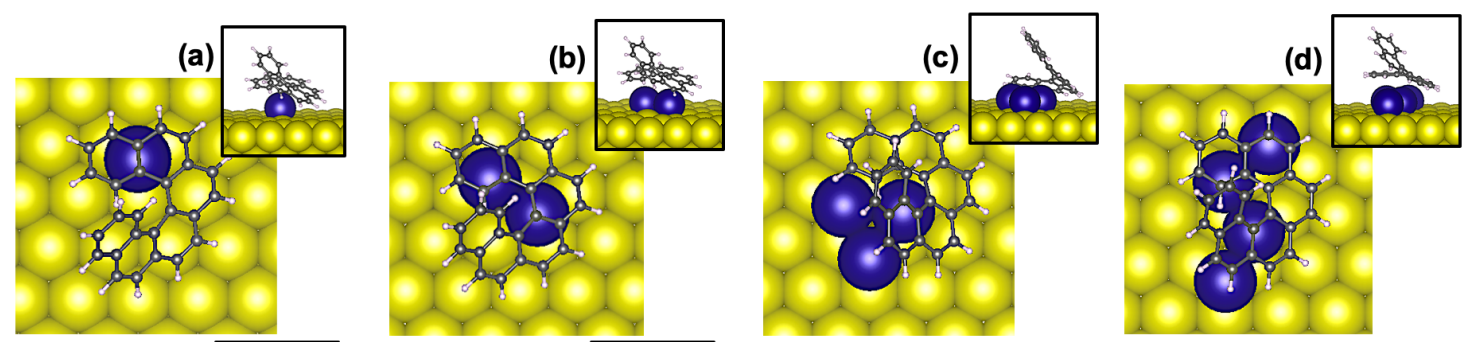

(e)

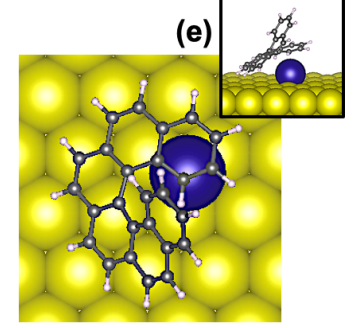

P-[6]H
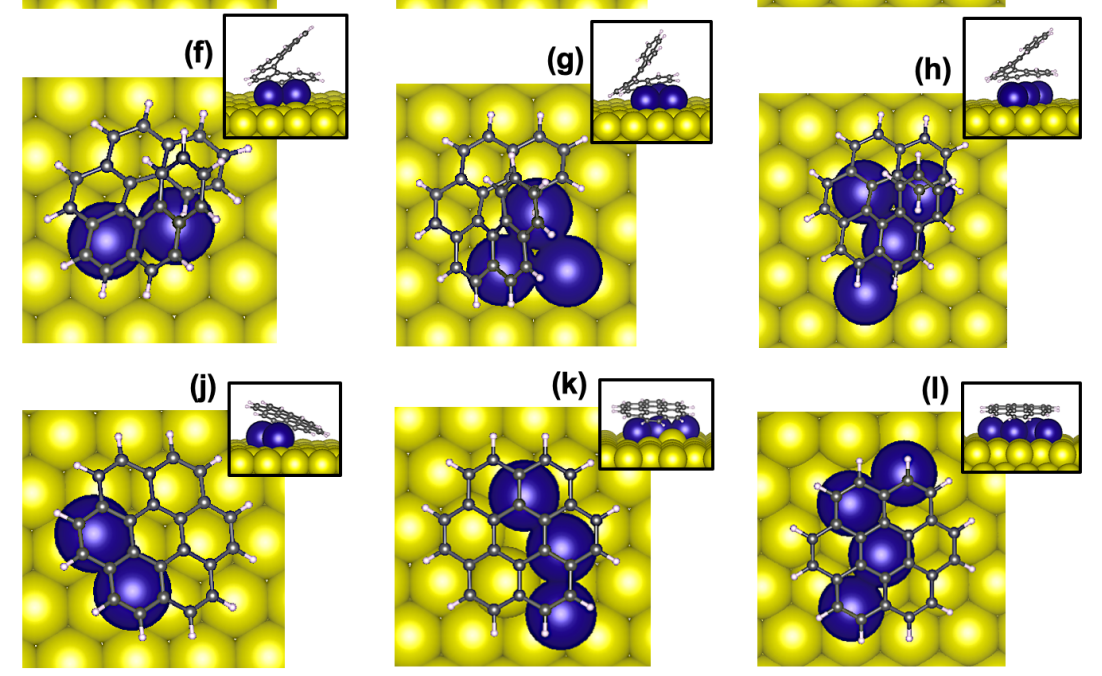
Figure 6. $M-[6] \mathrm{H}, P-[6] \mathrm{H}$, and $[6] \mathrm{C}$ adsorbed on $0,1,2,3$, and 4 adatom Au substrates. Regular $\mathrm{Au}$ substrate atoms are shown in yellow. Au adatoms are shown in blue. The full-width image is the top view of the system while the inset image is the side view. $M$-[6]H configurations adsorbed on surfaces with $0-4$ adatoms are shown in (a)-(d), respectively. $P$-[6]H configurations adsorbed on 0-4-adatom $\mathrm{Au}$ substrates are shown in (e)-(h), respectively. [6]C structures adsorbed on 0-4-adatom Au substrates are shown in (i)-(1), respectively.

In contrast to the unwieldy [6]C structure, helical $M-[6] \mathrm{H}$ and $P-[6] \mathrm{H}$ molecules adjust to the presence of the adatoms by coiling around them. The $M-[6] \mathrm{H}$ and $P-[6] \mathrm{H}$ enantiomers displayed similar binding energies regardless of the number of adatoms. The consistently weaker binding of [6]C and the stronger binding of helicenes to the defect substrates demonstrates the considerable effect adatoms have on the adsorption of PAHs. Conversely, adsorption of the PAHs often leads to reorganization of the adatom pattern, both in $0 \mathrm{~K}$ geometry optimizations, and in AIMD conditions (as seen in several panels of Figures 6 and 7). In fact, as shown in Table 1, helicenes consistently bind more strongly than [6]C on adatom-decorated substrates in both $\mathrm{Au}(111)$ and $\mathrm{Cu}(111)$-based systems. This behaviour is in contrast to the much stronger surface binding of [6]C on ideal substrates.

One striking trend seen in Table 1 is the consistent increase in [6] H binding energy on $\mathrm{Cu}$ with the number of adatoms. With each subsequent adatom added to the $\mathrm{Cu}$ surface, [6] $\mathrm{H}$ finds a way to interact more strongly with the substrate, demonstrating a remarkable versatility when it comes to monolayer stabilization in the presence of defects. Analysis of the ring height data in Figures 5(b) and (c) reflects this stronger helicene adsorption through overall lower adsorption distances on the adatom-decorated substrates than on ideal substrates. As was the case for [6]C, the strongest deformation is seen on the 2-adatom substrate, where the four rings closest to the surface are mainly flat. Binding strength is not correlated with minimum separation in these cases, however, as the 1-adatom complex, which experiences the weakest binding, also displays the smallest adatom-molecule separation, as well as a weaker flattening effect than on the ideal surface. Single adatoms bind to one ring or the junction of two rings, while the remaining 
backbone, too far from the underlying surface to effectively interact, coils away from it. On the other hand, helicenes bind strongest to the 3- and 4-adatom substrates considered here. The adatom structure is broad enough to support the basal region of the helicene, and the higher reactivity of the adatoms relative to the ideal (111) substrate leads to stronger flattening of the three rings adjacent to the adatoms, as well as stronger binding.

\section{Configurational sampling}

The similarity of the $M-[6] \mathrm{H}$ and $P$-[6]H binding energies, despite the structural complexity of the adsorption motif, prompted examination of the relative stability of various adsorbed conformations of $M-[6] \mathrm{H}$ and $P$-[6]H. Binding energies for the two enantiomers in different orientations on the 3-adatom surface are shown in Table 2.

Table 2. Binding energy $(\mathrm{eV})$ of rotated polyaromatic hydrocarbons adsorbed on 3-adatom $\mathrm{Cu}(111)$ substrates.

Binding energy (eV)

\begin{tabular}{cccccccccc}
\hline Adsorbate & $\mathbf{0}^{\circ *}$ & $\mathbf{3 0}^{\circ}$ & $\mathbf{6 0}^{\circ}$ & $\mathbf{9 0}^{\circ}$ & $\mathbf{1 2 0}^{\circ}$ & $\mathbf{1 5 0}^{\circ}$ & $\mathbf{1 8 0}^{\circ}$ & $\mathbf{2 4 0}^{\circ}$ & $\mathbf{3 0 0}^{\circ}$ \\
\hline$M-[6] \mathrm{H}$ & -2.40 & -1.87 & -1.95 & -2.20 & -2.05 & -2.42 & -2.40 & -2.26 & -2.23 \\
$P-[6] \mathrm{H}$ & -2.05 & -2.36 & -2.15 & -2.28 & -2.16 & -2.49 & -2.04 & -2.19 & -2.26
\end{tabular}

*The absolute values of the rotational angles do not have a specific physical meaning. They are provided for notational purposes and to indicate the relative (initial) orientations of the different conformations.

The stability of the monomer on the substrate varies depending on the initial rotational orientation of the monomer relative to the adatom structure. Not unexpectedly, the system lands in different minima on the potential energy surface, as the zero-Kelvin optimizations are unable to fully sample the conformational space. The most stable and least stable adsorbed conformations of the two enantiomers are presented in Figure 7. 


\section{Most stable}
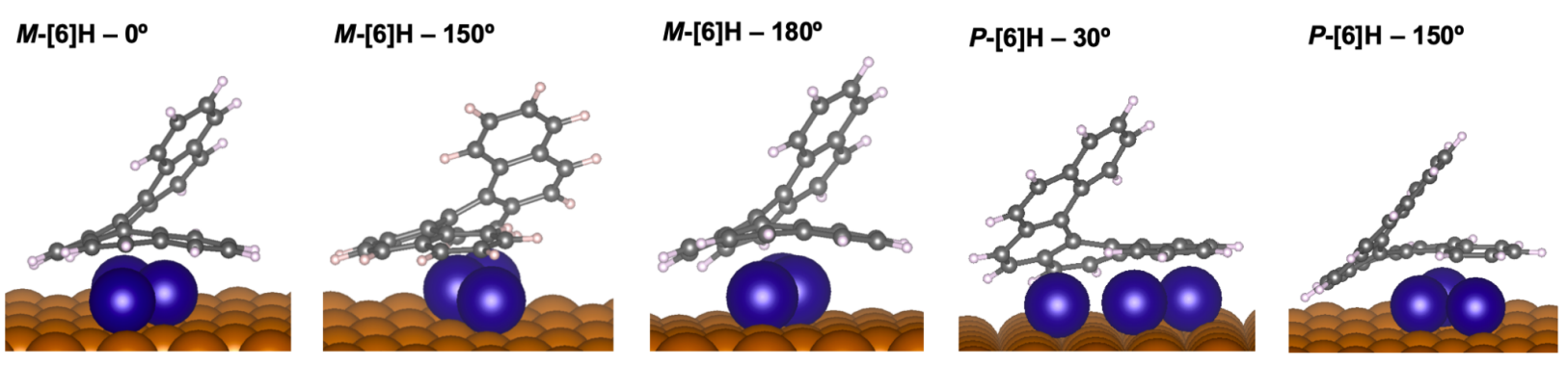

\section{Least stable}
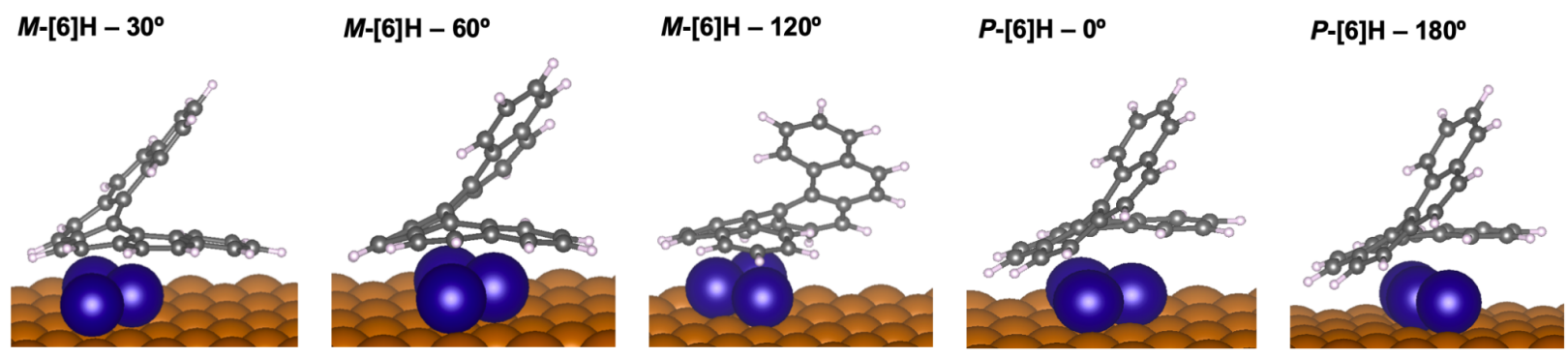

Figure 7. Low-energy and high-energy conformations of $M-[6] \mathrm{H}$ and $P-[6] \mathrm{H}$ on a $\mathrm{Cu}$ substrate functionalized with 3 adatoms. The three lowest energy (most stable) $M$-[6]H conformations (at $0^{\circ}, 150^{\circ}$, and $180^{\circ}$, see Table 2), and the two most stable $P$-[6]H conformations (at $30^{\circ}$ and $150^{\circ}$ ) are shown in the top row of the figure. The bottom row of the figure shows the three higher energy (least stable) conformations of $M$-[6]H (at $30^{\circ}, 60^{\circ}$, and $120^{\circ}$ ) and the two least stable $P$-[6]H conformations (at $0^{\circ}$ and $180^{\circ}$ ). Regular $\mathrm{Cu}$ substrate atoms are shown in orange. $\mathrm{Cu}$ adatoms are shown in blue.

The most stable conformations presented in Figure 7 share structural similarities: the helicene molecules are consistently centered over the adatoms in each of these low-energy arrangements. Favourable overlap of 3-4 adsorbate rings with all three adatoms leads to stronger interaction in these configurations, resulting in low molecule-surface distance. Although we did not study barrier heights, these structures would all be competitive at room temperature. Analysis of the molecular coiling, by quantifying ring heights as discussed in the Methods section, illustrates a different behaviour between the strongly-bound helicene molecules discussed here and those adsorbed on the ideal surface: on the ideal surface, rings near the surface flattened out, increasing 
coiling in the region of the molecule distant from the surface. On defect surfaces, the few adatoms have a smaller attractive "span," leading to less flattening of the rings near the substrate, and thus less coiling of the upper rings. As a result, the overall molecular heights (and thus the degree of coiling) of the strongly adsorbed helicene conformations are in the middle range of molecular heights for all the configurations considered here, despite their strong molecule-surface interactions (see Figure 8).
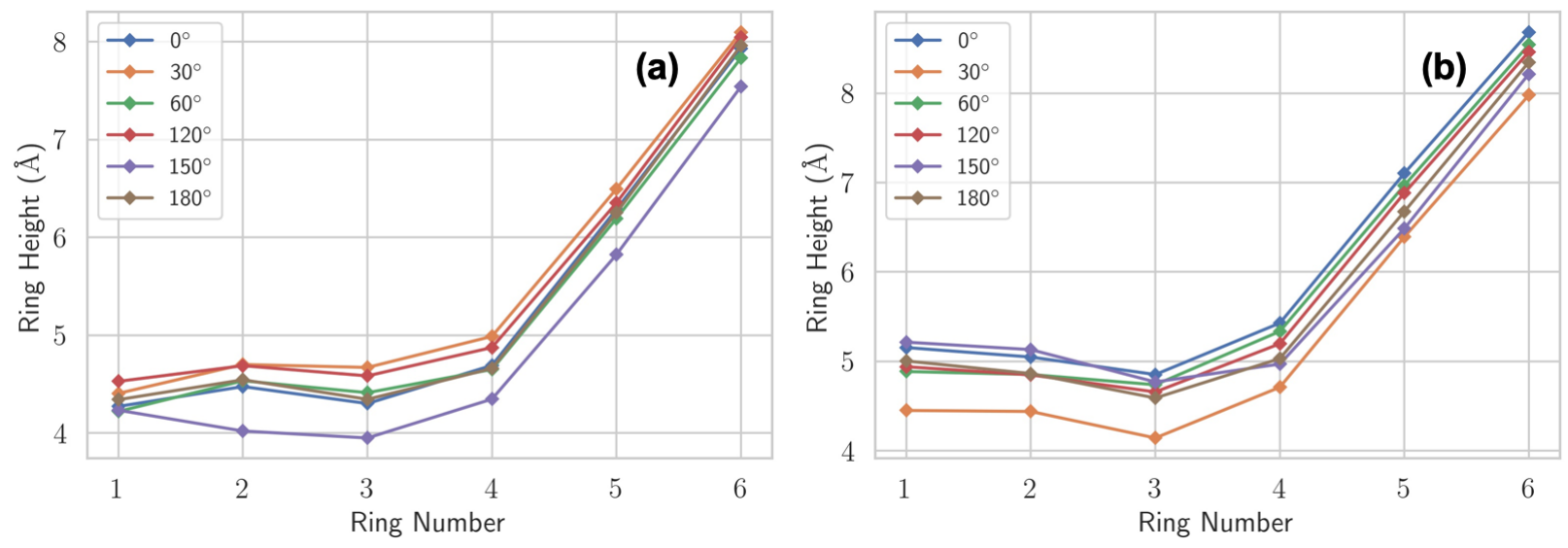

Figure 8. Individual ring heights for the sampled [6]H configurations on a 3-adatom substrate. Panel (a) shows the changes in ring height (from the adatom centers) for successive rings of $M$-[6]H in the series of configurations presented in Table 2. Panel (b) shows the changes in ring height for successive rings of $P-[6] \mathrm{H}$ in the series of configurations presented in Table 2.

In contrast, higher-energy configurations exhibit less efficient overlap: these initial structures converged to metastable states where 1-2 molecular rings overlap with 1-2 of the adatoms, leading to less efficient binding and larger molecule-surface separations. The structures were unable to achieve efficient stacking relative to the set of adatoms, instead landing asymmetrically onto one side of the adatom arrangement (as shown in Figure 7).

\section{AIMD simulations}

To develop a clearer picture of the complexity of the potential energy surface in the vicinity of the minima discussed above, AIMD calculations were performed for 2- and 3-adatom systems, starting from the most stable geometries discussed above. The additional thermal energy enables 
sampling of a larger configurational space around the minima used as initial structures. The most strongly bound configurations fundamentally retained the surface bonding structure, with kinetic energy distributed mainly into backbone distortions (see the SI movies M6H_3Cu_150deg.mov and $\mathrm{P} 6 \mathrm{H} \_3 \mathrm{Cu}$ _150deg.mov). Higher energy configurations explored a broader configurational space during the MD simulations, with reorganization of the adatom pattern (P6H_3Cu_180deg.mov) to support a larger section of the molecular backbone, or with a slide of the molecule towards the undecorated surface to satisfy interactions of the molecule with the flat substrate (as in $\mathrm{P} 6 \mathrm{H} \_3 \mathrm{Cu}_{-} 240$ deg.mov, for example), in addition to the molecule-adatom interactions.

\section{Lateral Dimers}

To explore adatom effects on enantiospecific lateral interactions, like and unlike [6]H dimers were situated side by side over 1 - and 3-adatom $\mathrm{Cu}$ substrates. In the initial dimer structures, one enantiomer was placed directly above the adatom, while the other enantiomer was placed nearby but above the portion of the substrate without the adatom. The configurational space sampled by helicene dimers on adatom-decorated substrates was even larger than what was available in the case of monomer binding, and standard algorithms for DFT-based structural optimization led to multiple metastable states close in geometry to the initial structures. To better understand the dimers' evolution when thermal energy was available, ab initio molecular dynamics calculations were carried out for several picoseconds. Representative snapshots were picked out from each simulation, and zero-Kelvin optimizations were carried out in order to determine binding energies comparable to those discussed in previous sections. The energies corresponding to the most stable configurations are reported in Table 3, and corresponding minimum energy structures are presented in Figure 9.

Table 3. Binding energy (eV) per molecule for hexahelicene dimers on a $\mathrm{Cu}$ substrate.

Binding energy (eV)

\begin{tabular}{ccccc}
\hline Surface & $M-[6] \mathrm{H}, P-[6] \mathrm{H}^{*}$ & $P-[6] \mathrm{H}, M-[6] \mathrm{H}$ & $M-[6] \mathrm{H}, M-[6] \mathrm{H}$ & $P-[6] \mathrm{H}, P-[6] \mathrm{H}$ \\
\hline 1-adatom & -2.20 & -2.46 & -2.29 & -2.25 \\
3-adatom & -2.53 & -2.55 & -2.28 & -
\end{tabular}


*The first molecule indicated in each pair was placed above the adatom in the initial structure.
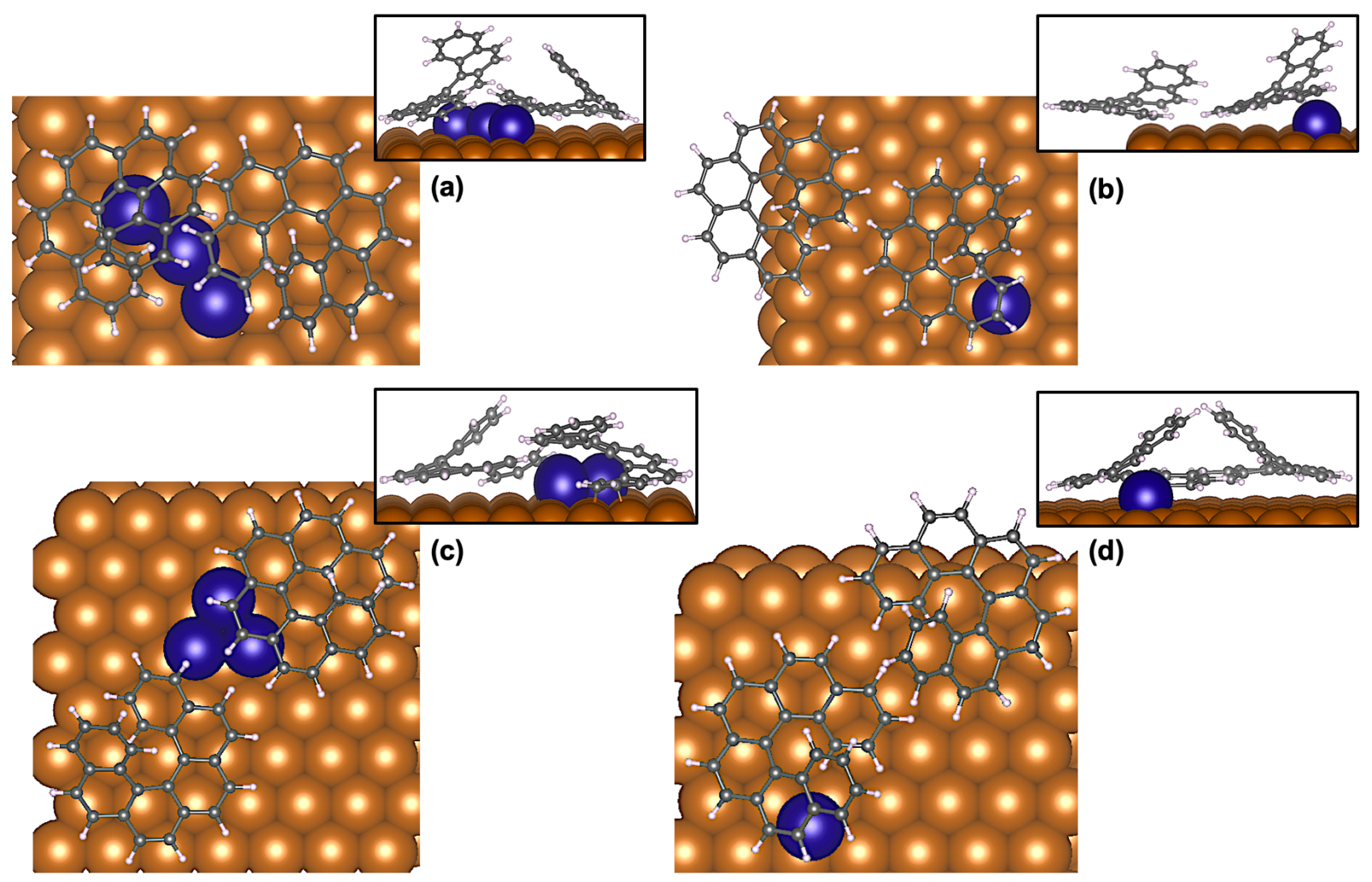

Figure 9. Structures of like and unlike dimers following DFT optimization. $\mathrm{Cu}$ adatoms are shown in blue. Panels (a) and (b) display a like dimer (the $M M$ dimer) on the 3-adatom and the 1-adatom surface, respectively. Panels (c) and (d) show stable configurations of the unlike dimer on the 3-adatom (the $M P$ dimer) and 1-adatom surfaces (the $P M$ dimer), respectively.

Surface interactions drove assembly in all of the lateral dimer calculations. Because of the limited defect footprint on 1-adatom surfaces, a single molecule was bound to the adatom in these cases. The second molecule bound to the adjacent ideal surface, experiencing the type of flattening observed in monomers deposited on ideal substrates. On 3-adatom substrates, both adsorbates were able to bind to the adatoms, and adatom migration is observed where this facilitates binding. As for monomers, 3-adatom binding was stronger than 1-adatom binding. Binding energies per molecule, however, are in the same range as monomer binding energies, 
suggesting that lateral interactions, including enantiospecific interactions, are negligible in this case.

\section{Conclusion}

This work details a computational investigation into the impact of adatom defects on the adsorption of polyaromatic hydrocarbons on coinage metal substrates. While on an ideal (111) metallic substrate, coronene establishes dispersive interactions over an extended area and thus binds more strongly than similar-footprint hexahelicenes. However, adatom defects in the surface reverse this trend. The out-of-plane coiling of helicenes and their increased molecular flexibility enabled better adaptability to the adatoms' presence. Helicenes were thus able to achieve more energetically favourable conformations than the planar coronene adsorbate by coiling around the adatoms and tilting so that part of the molecular backbone overlapped with the basal surface. The loss of contact area on adatom substrates is partly compensated by the higher reactivity of the lower-coordinated adatoms relative to the nine-coordinated atoms within the ideal surface. Hence, binding energies over clusters of 3 and 4 adatoms were similar to the extended surface binding for coronene. In the DFT-optimized monomers, rings 1-3 consistently remained relatively planar with respect to the underlying adatom-containing $\mathrm{Cu}$ substrate while rings 4-6 demonstrated considerable coiling. For dimers, surface interactions drive structure formation. For example, in one-adatom dimer systems, the sole adatom binds only one of the enantiomers, while the other enantiomer flattens as expected on the basal surface. By contrast, three-adatom systems evolve towards configurations where both molecules bind the reactive defect adatoms. These effects are observed in all systems considered here, regardless of chirality, which is an indication that chiral interactions take a secondary role to the increased surface reactivity. These findings improve our understanding of the impact surface irregularities have on the adsorption of PAHs on coinage metal substrates, and thus have relevance for further understanding of experimental results in these systems.

\section{Conflicts of interest}

There are no conflicts to declare. 


\section{Acknowledgements}

This research was conducted in part using the computational facilities managed by WestGrid (www.westgrid.ca) and Compute Canada (www.computecanada.ca). The authors are grateful to the National Science and Engineering Research Council for funding through the Discovery Grant and Undergraduate Student Research Award programs.

\section{Supporting information}

Optimized structures for all of the systems discussed here are provided as .xyz files. Movie files representing the time evolution of representative systems in the ab initio MD simulations are also provided online at ....

\section{References}

(1) Tuca, E.; Paci, I. Structural Analysis of Helicene Molecules Adsorbed on Symmetric Surfaces. Phys. Chem. Chem. Phys. 2019, 21 (18), 9189-9199. https://doi.org/10.1039/C9CP00284G.

(2) Yang, F.; Cheng, S.; Zhang, X.; Ren, X.; Li, R.; Dong, H.; Hu, W. 2D Organic Materials for Optoelectronic Applications. Adv. Mater. 2018, 30 (2), 1702415. https://doi.org/10.1002/adma.201702415.

(3) Otero, R.; Vázquez de Parga, A. L.; Gallego, J. M. Electronic, Structural and Chemical Effects of Charge-Transfer at Organic/Inorganic Interfaces. Surf. Sci. Rep. 2017, 72 (3), 105-145. https://doi.org/10.1016/j.surfrep.2017.03.001.

(4) Zielinska, A.; Leonowicz, M.; Li, H.; Nakanishi, T. Controlled Self-Assembly of Alkylated- $\pi$ Compounds for Soft Materials - Towards Optical and Optoelectronic Applications. Curr. Opin. Colloid Interface Sci. 2014, 19 (2), 131-139. https://doi.org/10.1016/j.cocis.2014.03.007.

(5) Moulin, E.; Busseron, E.; Giuseppone, N. CHAPTER 1 Self-Assembled Supramolecular Materials in Organic Electronics. 2014, 1-52. https://doi.org/10.1039/9781782626947-00001.

(6) Ariga, K.; Nishikawa, M.; Mori, T.; Takeya, J.; Shrestha, L. K.; Hill, J. P. Self-Assembly as a Key Player for Materials Nanoarchitectonics. Sci. Technol. Adv. Mater. 2019, 20 (1), 51-95. https://doi.org/10.1080/14686996.2018.1553108.

(7) Wang, Z.; Lin, H.; Zhang, X.; Li, J.; Chen, X.; Wang, S.; Gong, W.; Yan, H.; Zhao, Q.; Lv, W.; Gong, X.; Xiao, Q.; Li, F.; Ji, D.; Zhang, X.; Dong, H.; Li, L.; Hu, W. Revealing Molecular Conformation-Induced Stress at Embedded Interfaces of Organic Optoelectronic Devices by Sum Frequency Generation Spectroscopy. Sci. Adv. 2021, 7 (16), eabf8555. https://doi.org/10.1126/sciadv.abf8555.

(8) Lu, F.; Nakanishi, T. Alkyl-\$luppi\$engineering in State Control toward Versatile Optoelectronic Soft Materials. Sci. Technol. Adv. Mater. 2015, 16 (1), 014805. 
https://doi.org/10.1088/1468-6996/16/1/014805.

(9) Boles, M. A.; Engel, M.; Talapin, D. V. Self-Assembly of Colloidal Nanocrystals: From Intricate Structures to Functional Materials. Chem. Rev. 2016, 116 (18), 11220-11289. https://doi.org/10.1021/acs.chemrev.6b00196.

(10) Ferrari, A. C.; Bonaccorso, F.; Fal'ko, V.; Novoselov, K. S.; Roche, S.; Bøggild, P.; Borini, S.; Koppens, F. H. L.; Palermo, V.; Pugno, N.; Garrido, J. A.; Sordan, R.; Bianco, A.; Ballerini, L.; Prato, M.; Lidorikis, E.; Kivioja, J.; Marinelli, C.; Ryhänen, T.; Morpurgo, A.; Coleman, J. N.; Nicolosi, V.; Colombo, L.; Fert, A.; Garcia-Hernandez, M.; Bachtold, A.; Schneider, G. F.; Guinea, F.; Dekker, C.; Barbone, M.; Sun, Z.; Galiotis, C.;

Grigorenko, A. N.; Konstantatos, G.; Kis, A.; Katsnelson, M.; Vandersypen, L.; Loiseau, A.; Morandi, V.; Neumaier, D.; Treossi, E.; Pellegrini, V.; Polini, M.; Tredicucci, A.; Williams, G. M.; Hee Hong, B.; Ahn, J.-H.; Min Kim, J.; Zirath, H.; van Wees, B. J.; van der Zant, H.; Occhipinti, L.; Di Matteo, A.; Kinloch, I. A.; Seyller, T.; Quesnel, E.; Feng, X.; Teo, K.; Rupesinghe, N.; Hakonen, P.; Neil, S. R. T.; Tannock, Q.; Löfwander, T.; Kinaret, J. Science and Technology Roadmap for Graphene, Related Two-Dimensional Crystals, and Hybrid Systems. Nanoscale 2015, 7 (11), 4598-4810. https://doi.org/10.1039/C4NR01600A.

(11) Ariga, K.; Mori, T.; Kitao, T.; Uemura, T. Supramolecular Chiral Nanoarchitectonics. Adv. Mater. 2020, 32 (41), 1905657. https://doi.org/10.1002/adma.201905657.

(12) Liu, W.; Tkatchenko, A.; Scheffler, M. Modeling Adsorption and Reactions of Organic Molecules at Metal Surfaces. Acc. Chem. Res. 2014, 47 (11), 3369-3377. https://doi.org/10.1021/ar500118y.

(13) Ernst, K.-H. Stereochemical Recognition of Helicenes on Metal Surfaces. Acc. Chem. Res. 2016, 49 (6), 1182-1190. https://doi.org/10.1021/acs.accounts.6b00110.

(14) Newman, M. S.; Darlak, R. S.; Tsai, L. L. Optical Properties of Hexahelicene. J. Am. Chem. Soc. 1967, 89 (24), 6191-6193. https://doi.org/10.1021/ja01000a034.

(15) Isla, H.; Crassous, J. Helicene-Based Chiroptical Switches. Comptes Rendus Chim. 2016, 19 (1), 39-49. https://doi.org/10.1016/j.crci.2015.06.014.

(16) Gingras, M. One Hundred Years of Helicene Chemistry. Part 1: Non-Stereoselective Syntheses of Carbohelicenes. Chem. Soc. Rev. 2013, 42 (3), 968-1006. https://doi.org/10.1039/C2CS35154D.

(17) Fasel, R.; Parschau, M.; Ernst, K.-H. Chirality Transfer from Single Molecules into Self-Assembled Monolayers. Angew. Chem. Int. Ed. 2003, 42 (42), 5178-5181. https://doi.org/10.1002/anie.200352232.

(18) Hatakeyama, T.; Hashimoto, S.; Oba, T.; Nakamura, M. Azaboradibenzo[6]Helicene: Carrier Inversion Induced by Helical Homochirality. J. Am. Chem. Soc. 2012, 134 (48), 19600-19603. https://doi.org/10.1021/ja310372f.

(19) Kaseyama, T.; Furumi, S.; Zhang, X.; Tanaka, K.; Takeuchi, M. Hierarchical Assembly of a Phthalhydrazide-Functionalized Helicene. Angew. Chem. Int. Ed. 2011, 50 (16), 3684-3687. https://doi.org/10.1002/anie.201007849.

(20) Seibel, J.; Zoppi, L.; Ernst, K.-H. 2D Conglomerate Crystallization of Heptahelicene. Chem. Commun. 2014, 50 (63), 8751-8753. https://doi.org/10.1039/C4CC03574G.

(21) Hill, J. P.; Jin, W.; Kosaka, A.; Fukushima, T.; Ichihara, H.; Shimomura, T.; Ito, K.; Hashizume, T.; Ishii, N.; Aida, T. Self-Assembled Hexa-Peri-Hexabenzocoronene Graphitic Nanotube. Science 2004, 304 (5676), 1481-1483. 
https://doi.org/10.1126/science.1097789.

(22) Stankovich, S.; Dikin, D. A.; Dommett, G. H. B.; Kohlhaas, K. M.; Zimney, E. J.; Stach, E. A.; Piner, R. D.; Nguyen, S. T.; Ruoff, R. S. Graphene-Based Composite Materials. Nature 2006, 442 (7100), 282-286. https://doi.org/10.1038/nature04969.

(23) Rapacioli, M.; Calvo, F.; Spiegelman, F.; Joblin, C.; Wales, D. J. Stacked Clusters of Polycyclic Aromatic Hydrocarbon Molecules. J. Phys. Chem. A 2005, 109 (11), 2487-2497. https://doi.org/10.1021/jp046745z.

(24) Stöhr, M.; Boz, S.; Schär, M.; Nguyen, M.-T.; Pignedoli, C. A.; Passerone, D.; Schweizer, W. B.; Thilgen, C.; Jung, T. A.; Diederich, F. Self-Assembly and Two-Dimensional Spontaneous Resolution of Cyano-Functionalized [7]Helicenes on $\mathrm{Cu}(111)$. Angew. Chem. Int. Ed. 2011, 50 (42), 9982-9986. https://doi.org/10.1002/anie.201102627.

(25) Rahe, P.; Nimmrich, M.; Greuling, A.; Schütte, J.; Stará, I. G.; Rybáček, J.; Huerta-Angeles, G.; Starý, I.; Rohlfing, M.; Kühnle, A. Toward Molecular Nanowires Self-Assembled on an Insulating Substrate: Heptahelicene-2-Carboxylic Acid on Calcite (1014). J. Phys. Chem. C 2010, 114 (3), 1547-1552. https://doi.org/10.1021/jp911287p.

(26) Ernst, K.-H.; Kuster, Y.; Fasel, R.; McFadden, C. F.; Ellerbeck, U. Adsorption of Helical Aromatic Molecules: Heptahelicene on Ni(111). Surf. Sci. 2003, 530 (3), 195-202. https://doi.org/10.1016/S0039-6028(03)00489-8.

(27) Ernst, K.-H.; Neuber, M.; Grunze, M.; Ellerbeck, U. NEXAFS Study on the Orientation of Chiral P-Heptahelicene on Ni(100). J. Am. Chem. Soc. 2001, 123 (3), 493-495. https://doi.org/10.1021/ja003262+.

(28) Ernst, K.-H.; Kuster, Y.; Fasel, R.; Müller, M.; Ellerbeck, U. Two-Dimensional Separation of [7]Helicene Enantiomers on $\mathrm{Cu}(111)$. Chirality 2001, 13 (10), 675-678. https://doi.org/10.1002/chir.10006.

(29) Seibel, J.; Parschau, M.; Ernst, K.-H. Two-Dimensional Crystallization of Enantiopure and Racemic Heptahelicene on $\mathrm{Ag}(111)$ and $\mathrm{Au}(111)$. J. Phys. Chem. C 2014, 118 (50), 29135-29141. https://doi.org/10.1021/jp504673g.

(30) Rybáček, J.; Huerta-Angeles, G.; Kollárovič, A.; Stará, I. G.; Starý, I.; Rahe, P.; Nimmrich, M.; Kühnle, A. Racemic and Optically Pure Heptahelicene-2-Carboxylic Acid: Its Synthesis and Self-Assembly into Nanowire-Like Aggregates. Eur. J. Org. Chem. 2011, 2011 (5), 853-860. https://doi.org/10.1002/ejoc.201001110.

(31) Cao, H.; Waghray, D.; Knoppe, S.; Dehaen, W.; Verbiest, T.; De Feyter, S. Tailoring Atomic Layer Growth at the Liquid-Metal Interface. Nat. Commun. 2018, 9 (1), 4889. https://doi.org/10.1038/s41467-018-07381-w.

(32) Bartels, L. Tailoring Molecular Layers at Metal Surfaces. Nat. Chem. 2010, 2 (2), 87-95. https://doi.org/10.1038/nchem.517.

(33) García, A.; Papior, N.; Akhtar, A.; Artacho, E.; Blum, V.; Bosoni, E.; Brandimarte, P.; Brandbyge, M.; Cerdá, J. I.; Corsetti, F.; Cuadrado, R.; Dikan, V.; Ferrer, J.; Gale, J.; García-Fernández, P.; García-Suárez, V. M.; García, S.; Huhs, G.; Illera, S.; Korytár, R.; Koval, P.; Lebedeva, I.; Lin, L.; López-Tarifa, P.; Mayo, S. G.; Mohr, S.; Ordejón, P.; Postnikov, A.; Pouillon, Y.; Pruneda, M.; Robles, R.; Sánchez-Portal, D.; Soler, J. M.; Ullah, R.; Yu, V. W.; Junquera, J. Siesta: Recent Developments and Applications. J. Chem. Phys. 2020, 152 (20), 204108. https://doi.org/10.1063/5.0005077.

(34) Soler, J. M.; Artacho, E.; Gale, J. D.; García, A.; Junquera, J.; Ordejón, P.; Sánchez-Portal, D. The SIESTA Method Forab Initioorder-Nmaterials Simulation. J. Phys. Condens. 
Matter 2002, 14 (11), 2745-2779. https://doi.org/10.1088/0953-8984/14/11/302.

(35) Perdew, J. P.; Burke, K.; Ernzerhof, M. Generalized Gradient Approximation Made Simple. Phys. Rev. Lett. 1996, 77 (18), 3865-3868.

https://doi.org/10.1103/PhysRevLett.77.3865.

(36) Tkatchenko, A.; Scheffler, M. Accurate Molecular Van Der Waals Interactions from Ground-State Electron Density and Free-Atom Reference Data. Phys. Rev. Lett. 2009, 102 (7), 073005. https://doi.org/10.1103/PhysRevLett.102.073005.

(37) Grimme, S. Semiempirical GGA-type density functional constructed with a long-range dispersion correction. J. Comput. Chem. 2006, 27 (15), 1787-1799. https://doi.org/10.1002/jcc.20495.

(38) Grimme, S.; Antony, J.; Ehrlich, S.; Krieg, H. A Consistent and Accurate Ab Initio Parametrization of Density Functional Dispersion Correction (DFT-D) for the 94 Elements H-Pu. J. Chem. Phys. 2010, 132 (15), 154104. https://doi.org/10.1063/1.3382344.

(39) Boys, S. F.; Bernardi, F. The Calculation of Small Molecular Interactions by the Differences of Separate Total Energies. Some Procedures with Reduced Errors. Mol. Phys. 1970, 19 (4), 553-566. https://doi.org/10.1080/00268977000101561.

(40) Simon, S.; Duran, M.; Dannenberg, J. J. How Does Basis Set Superposition Error Change the Potential Surfaces for Hydrogen-bonded Dimers? J. Chem. Phys. 1996, 105 (24), 11024-11031. https://doi.org/10.1063/1.472902.

(41) Ting, E. C. M.; Popa, T.; Paci, I. Surface-Site Reactivity in Small-Molecule Adsorption: A Theoretical Study of Thiol Binding on Multi-Coordinated Gold Clusters. Beilstein J. Nanotechnol. 2016, 7 (1), 53-61. https://doi.org/10.3762/bjnano.7.6.

(42) Chapman, C. R. L.; Ting, E. C. M.; Kereszti, A.; Paci, I. Self-Assembly of Cysteine Dimers at the Gold Surface: A Computational Study of Competing Interactions. J. Phys. Chem. C 2013, 117 (38), 19426-19435. https://doi.org/10.1021/jp405478n.

(43) Vasileff, A.; Xu, C.; Jiao, Y.; Zheng, Y.; Qiao, S.-Z. Surface and Interface Engineering in Copper-Based Bimetallic Materials for Selective CO2 Electroreduction. Chem 2018, 4 (8), 1809-1831. https://doi.org/10.1016/j.chempr.2018.05.001.

(44) Hammer, B.; Nørskov, J. K. Theoretical Surface Science and Catalysis - Calculations and Concepts. In Advances in Catalysis; Impact of Surface Science on Catalysis; Academic Press, 2000; Vol. 45, pp 71-129. https://doi.org/10.1016/S0360-0564(02)45013-4.

(45) Norskov, J. K.; Abild-Pedersen, F.; Studt, F.; Bligaard, T. Density Functional Theory in Surface Chemistry and Catalysis. Proc. Natl. Acad. Sci. 2011, 108 (3), 937-943. https://doi.org/10.1073/pnas.1006652108. 